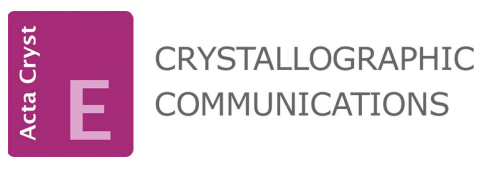

ISSN 2056-9890

Received 1 September 2016

Accepted 6 September 2016

Edited by A. Van der Lee, Université de Montpellier II, France

Keywords: crystal structure; powder diffraction; sodium hafnium disilicate.

CCDC reference: 1502957

Supporting information: this article has supporting information at journals.iucr.org/e

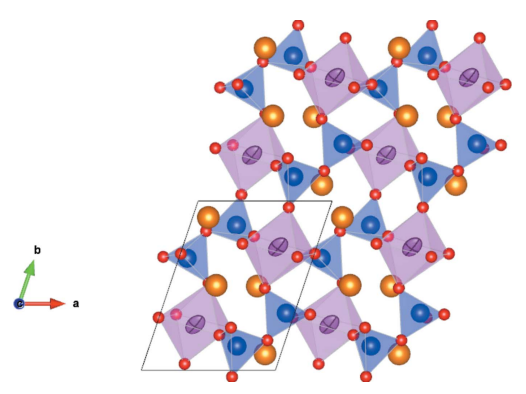

OPEN $\odot$ ACCESS

\section{Crystal structure of $\mathrm{Na}_{2} \mathrm{HfSi}_{2} \mathrm{O}_{7}$ by Rietveld refinement}

\author{
Nicolas Massoni $^{\mathrm{a} *}$ and Pierrick Chevreux ${ }^{\mathrm{a}, \mathrm{b}}$ \\ ${ }^{a}$ CEA, DEN, DTCD, Marcoule, BP17171, F-30207 Bagnols sur Ceze, France, and ${ }^{\mathbf{b}}$ CRPG, CNRS UMR-5873, Université \\ de Lorraine, BP 20, F-54501 Vandoeuvre les Nancy cedex, France. *Correspondence e-mail: nicolas.massoni@cea.fr
}

The structure of triclinic disodium hafnium disilicate, $\mathrm{Na}_{2} \mathrm{HfSi}_{2} \mathrm{O}_{7}$, has been determined by laboratory powder X-ray diffraction and refined by the Rietveld refinement. The structure is a framework made of alternate layers of $\mathrm{HfO}_{6}$ octahedra and $\mathrm{SiO}_{4}$ tetrahedra linked by common $\mathrm{O}$ atoms. Sodium atoms are located in the voids of the framework, aligned into tunnels along the [010] direction. $\mathrm{Na}_{2} \mathrm{HfSi}_{2} \mathrm{O}_{7}$ is isostructural with the parakeldyshite $\mathrm{Na}_{2} \mathrm{ZrSi}_{2} \mathrm{O}_{7}$ phase.

\section{Chemical context}

Laboratory work in order to explore the chemistry of compounds with radioactive elements such as actinides is difficult because of the emission of ionizing radiation. To overcome this problem, these radionuclides are often replaced by a stable element having similar properties as the radioactive element, for instance by using elements with a similar ionic radius or with the same oxidation state. Hence actinides are often replaced by neodymium, zirconium, europium, or hafnium (Ramsey et al., 1995). The reactivity of uranium with an $\mathrm{Na}-\mathrm{Si}-\mathrm{O}$ glass at high temperatures was thus simulated by using hafnium instead of uranium. We have obtained samples with different phases among which was a sodium hafnium disilicate, similar to the sodium zirconium silicate already observed in a similar glass (Plaisted et al., 1999). The structure of the sodium hafnium disilicate is discussed in this paper.

\section{Structural commentary}

The $\mathrm{Na}_{2} \mathrm{HfSi}_{2} \mathrm{O}_{7}$ phase is isostructural with the parakeldyshite phase (Voronkov et al., 1970; Fleischer et al., 1979). As reported in Table 1, the cell parameters of the $\mathrm{Na}_{2} \mathrm{HfSi}_{2} \mathrm{O}_{7}$ phase are slightly smaller than those of parakeldyshite, and the volume of the cell is $0.8 \%$ smaller. For the $\mathrm{Na}_{2} \mathrm{HfSi}_{2} \mathrm{O}_{7}$ phase, the $\mathrm{Hf} \mathrm{O}_{6}$ octahedral and the $\mathrm{Si} 2 \mathrm{O}_{4}$ tetrahedral volumes are about the same as the analogous $\mathrm{Zr}$ octahedral and $\mathrm{Si}$ tetrahedral volumes in parakeldyshite. The $\mathrm{Si}_{4} \mathrm{O}_{4}$ tetrahedral volume of the $\mathrm{Na}_{2} \mathrm{HfSi}_{2} \mathrm{O}_{7}$ phase is about $5 \%$ smaller than that in parakeldyshite. It is thus in the latter tetrahedron that the bond lengths differ significantly whereas the other bond lengths are quite similar in both phases. The sodium coordination polyhedral volumes are quite similar in volume for the two phases, about $30.1 \AA^{3}$. A polyhedral view of the $\mathrm{Na}_{2} \mathrm{HfSi}_{2} \mathrm{O}_{7}$ structure is given in Fig. 1. The $\mathrm{Na}_{2} \mathrm{ZrSi}_{2} \mathrm{O}_{7}$ phase is capable of ion exchange on the sodium site thanks to the sufficient dimension of the sodium tunnels in the [010] 
Table 1

Cell parameters, selected distances $(\AA)$, angles $\left(^{\circ}\right)$ and volumes $\left(\AA^{3}\right)$ for the title phase compared to parakeldyshite.

Cell parameters of $\mathrm{Na}_{2} \mathrm{ZrSi}_{2} \mathrm{O}_{7}$ are from Ferreira et al. (2001).

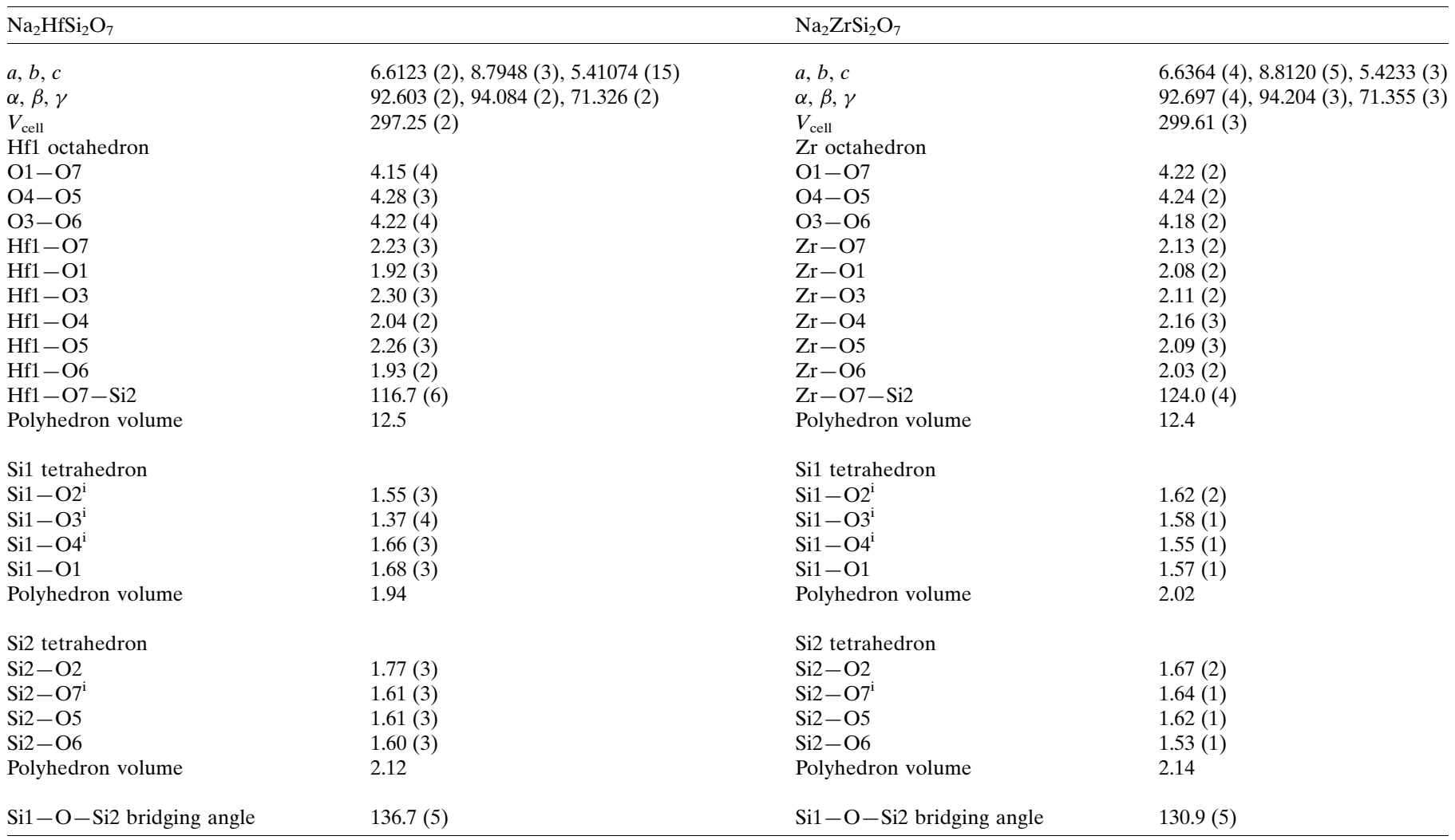

Symmetry code: (i) $-x,-y,-z$.

direction (Kostov-Kytin et al., 2008). Since these dimensions are the same in both phases, ion exchange should also be possible in the $\mathrm{Na}_{2} \mathrm{HfSi}_{2} \mathrm{O}_{7}$ phase. A numerical comparison of the structures of the parakeldyshite and the $\mathrm{Na}_{2} \mathrm{HfSi}_{2} \mathrm{O}_{7}$ phase
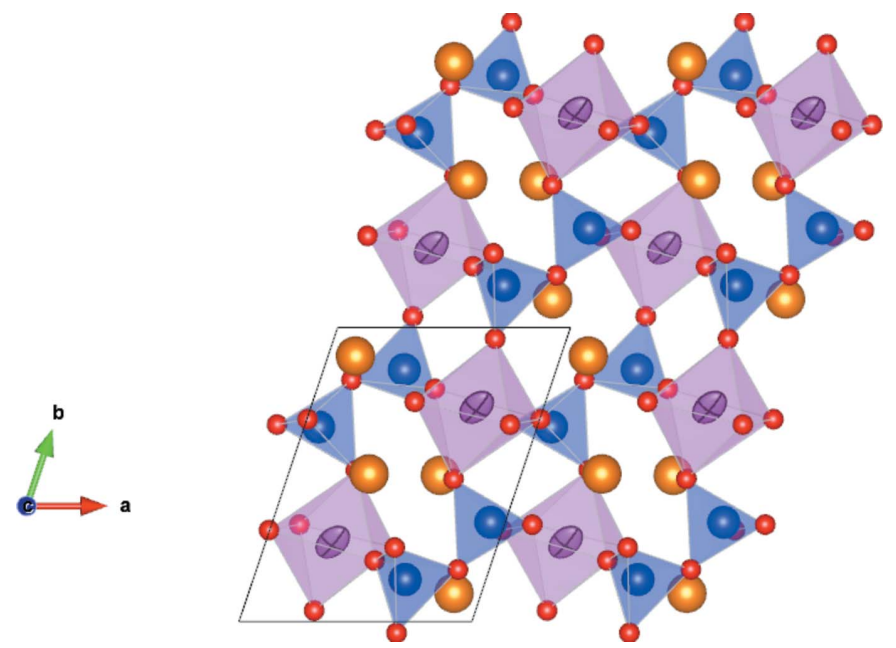

Figure 1

Polyhedral representation of the $\mathrm{Na}_{2} \mathrm{HfSi}_{2} \mathrm{O}_{7}$ phase with $\mathrm{SiO}_{4}$ units (blue), $\mathrm{HfO}_{6}$ units (green) and sodium (yellow) with displacement ellipsoids drawn at the $99 \%$ probability level. was performed with COMPSTRU (de la Flor et al., 2016). The structures' similarities were estimated by different parameters such as the measure of similarity $\Delta$ (Bergerhoff et al., 1999). This parameter was determined to be 0.018 for a maximum distance between paired atoms of $1 \AA$, indicating that structures are effectively isostructural. Since hafnium simulates uranium, the existence of the $\mathrm{Na}_{2} \mathrm{USi}_{2} \mathrm{O}_{7}$ phase can also be supposed.

\section{Database survey}

The crystal chemistry of zirconosilicates can be described in terms of an $M T$ framework with $M \mathrm{O}_{6}$ octahedra and $T \mathrm{O}_{4}$ tetrahedra $(M=\mathrm{Zr}, T=\mathrm{Si}$; Ilyushin \& Blatov, 2002). The voids in the $M T$ framework are filled with alkaline or alkaline earth elements coordinated in an eight-vertex polyhedron. The crystal system of sodium zirconosilicates can vary from triclinic $\left(\mathrm{Na}_{2} \mathrm{ZrSi}_{2} \mathrm{O}_{7}\right)$ to monoclinic $\left(\mathrm{Na}_{2} \mathrm{ZrSi}_{4} \mathrm{O}_{11}\right)$ or trigonal $\left(\mathrm{Na}_{8} \mathrm{ZrSi}_{6} \mathrm{O}_{18}\right)$. If we focus on the chemistry of zirconosilicates with $\mathrm{Si}_{2} \mathrm{O}_{7}$ diortho groups and their analogs (Pekov et al., 2007), the triclinic phase is privileged such as the parakeldyshite $\mathrm{Na}_{2} \mathrm{ZrSi}_{2} \mathrm{O}_{7}$ phase (Ferreira et al., 2001) or the keldyshite $(\mathrm{Na}, \mathrm{H})_{2} \mathrm{ZrSi}_{2} \mathrm{O}_{7}$ phase (Khalilov et al., 1978). The potassium analogue, however, is monoclinic as in the case of khibinskite $\mathrm{K}_{2} \mathrm{ZrSi}_{2} \mathrm{O}_{7}$ (Chernov et al., 1970; Nosyrev et al., 1976). 


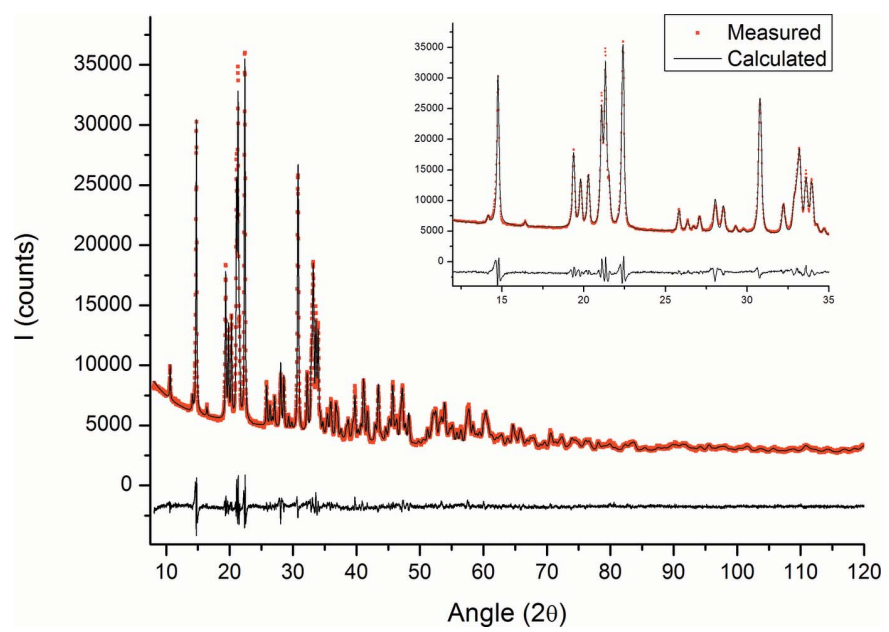

Figure 2

Comparison of observed (red squares) and calculated (solid line) intensities for $\mathrm{Na}_{2} \mathrm{HfSi}_{2} \mathrm{O}_{7}$. The difference pattern appears below. Inset: focus on the $12-35^{\circ} 2 \theta$ range.

\section{Synthesis and crystallization}

The synthesis of sodium hafnium disilicate was based on the two-step synthesis protocol of parakeldyshite $\mathrm{Na}_{2} \mathrm{ZrSi}_{2} \mathrm{O}_{7}$ ( $\mathrm{Lin}$ et al., 1999; Ferreira et al., 2001). The first step was the synthesis of the $\mathrm{Hf}-$ petarasite phase $\mathrm{Na}_{5} \mathrm{Zr}_{2} \mathrm{Si}_{6} \mathrm{O}_{18}(\mathrm{Cl} \cdot \mathrm{OH})_{2} \cdot-$ $\mathrm{H}_{2} \mathrm{O}$ with zirconium totally substituted by hafnium. Adequate quantities of sodium silicate solution $\left(27 \% \mathrm{SiO}_{2}, 8 \% \mathrm{Na}_{2} \mathrm{O}\right)$, sodium chloride, hafnium chloride, potassium chloride, sodium hydroxide and water were mixed thoroughly in a polytetrafluoroethylene (PTFE) vessel at room temperature for 30 minutes. A gel was obtained with a $\mathrm{pH}$ value around 13 . The PTFE vessel was put in a Parr digestion apparatus for a hydrothermal synthesis over 10 days at $523 \mathrm{~K}$. The resulting powder was washed, filtered, and dried overnight at $393 \mathrm{~K}$. In spite of the drying process, the powder was still hydrated. Powder X-ray diffraction showed the compound to be isostructural to petarasite. The second step was the calcination of Hf-petarasite over $15 \mathrm{~h}$ at $1373 \mathrm{~K}$ under air which lead to a white powder. SEM observation of the powder showed large grains with $\mathrm{Na}, \mathrm{Hf}, \mathrm{Si}$ and $\mathrm{O}$ and smaller grains with supplementary K. The chemical composition of the major phase was determined by EDS to have the following stoichiometry $\mathrm{Na}_{1.7 \pm 0.2} \mathrm{Hf}_{1.0} \mathrm{Si}_{2.3 \pm 0.1} \mathrm{O}_{7.3 \pm 0.9}$ as compared to the theoretical stoichiometry of $\mathrm{Na}_{2} \mathrm{HfSi}_{2} \mathrm{O}_{7}$. Thus the major phase is very close to the expected one. The sample was analysed by differential thermal analysis to determine its melting point. There was no thermal event indicating a melting until $1623 \mathrm{~K}$ and the sample was still in powder form. The $\mathrm{Na}_{2} \mathrm{HfSi}_{2} \mathrm{O}_{7}$ phase therefore has a higher melting point than parakeldyshite which is below $1523 \mathrm{~K}$ (Ferreira et al., 2001).

\section{Refinement}

Crystal data, data collection and structure refinement details are summarized in Table 2. Observed and calculated intensities for $\mathrm{Na}_{2} \mathrm{HfSi}_{2} \mathrm{O}_{7}$ are shown in Fig. 2 along with the
Table 2

Experimental details.

\begin{tabular}{ll}
\hline Crystal data & \\
Chemical formula & $\mathrm{Na}_{2} \mathrm{HfSi}_{2} \mathrm{O}_{7}$ \\
$M_{\mathrm{r}}$ & 392.6 \\
Crystal system, space group & Triclinic, $P \overline{1}$ \\
Temperature $(\mathrm{K})$ & 293 \\
$a, b, c(\AA)$ & $6.6123(2), 8.7948(3), 5.41074(15)$ \\
$\alpha, \beta, \gamma\left({ }^{\circ}\right)$ & $92.603(2), 94.0843(18)$, \\
$V\left(\AA^{3}\right)$ & $71.3262(18)$ \\
$Z$ & $297.25(2)$ \\
Radiation type & 2 \\
Specimen shape, size $(\mathrm{mm})$ & $\mathrm{Cu} K \alpha_{1}, \lambda=1.540562,1.544390 \AA$ \\
& Flat sheet, $25 \times 25$ \\
Data collection & \\
Diffractometer & Panalytical XPert MPD Pro \\
Specimen mounting & Packed powder pellet \\
Data collection mode & Reflection \\
Scan method & Step \\
$2 \theta$ values $\left({ }^{\circ}\right)$ & $2 \theta_{\min }=8.0132 \theta_{\max }=120.0132 \theta_{\text {step }}$ \\
& $=0.017$ \\
Refinement & \\
$R$ factors and goodness of fit & $R_{\mathrm{p}}=0.024, R_{\mathrm{wp}}=0.032$, \\
& $R_{\text {exp }}=0.015, R(F)=0.024$, \\
& $\chi^{2}=4.973$ \\
No. of parameters & 67 \\
\hline
\end{tabular}

Computer programs: X'Pert Data Collector (PANalytical, 2011), JANA2006 (Petříček et al., 2014), VESTA (Momma \& Izumi, 2011) and publCIF (Westrip, 2010).

difference pattern. The reliability factors of the refinement were quite poor because of an amorphous bump attributed to the second minor phase. Hence the reliability factors were negatively impacted. The isotropic ADP's of the oxygen atoms were constrained to be equal in volume in order to avoid a slightly negative ADP value on O5. The residual electron density is about $2.4 \mathrm{e}^{3}$, which is less than $10 \%$ of the electron density of a Hf atom. The occupancies of all atoms were fixed to unity.

\section{Acknowledgements}

NM would like to thank Professor Philippe Deniard (Institut des Matériaux de Nantes) for his continuous support of the Rietveld refinements. The authors gratefully acknowledge AREVA and ANDRA for their financial support of this work.

\section{References}

Bergerhoff, G., Berndt, M., Brandenburg, K. \& Degen, T. (1999). Acta Cryst. B55, 147-156.

Chernov, A. N., Maksimov, B. A., Ilyukhin, V. V. \& Belov, N. V. (1970). Dokl. Akad. Nauk SSSR, 193, 1293-1296.

Ferreira, P., Ferreira, A., Rocha, J. \& Soares, M. R. (2001). Chem. Mater. 13, 355-363.

Fleischer, M., Chao, G. Y. \& Mandarino, J. A. (1979). Am. Mineral. 64, 652-659.

Flor, G. de la, Orobengoa, D., Tasci, E., Perez-Mato, J. M. \& Aroyo, M. I. (2016). J. Appl. Cryst. 49, 653-664.

Ilyushin, G. D. \& Blatov, V. A. (2002). Acta Cryst. B58, 198-218.

Khalilov, A. D., Khomyakov, A. P. \& Makhmudov, S. A. (1978). Dokl. Akad. Nauk SSSR, 238, 573-575.

Kostov-Kytin, V., Fujiwara, K., Nakayama, N. \& Nikolova, R. (2008). Proc. Bulg. Geol. Soc. pp. 13-14. 
Lin, Z., Rocha, J., Ferreira, P., Thursfield, A., Agger, J. R. \& Anderson, M. W. (1999). J. Phys. Chem. B, 103, 957-963.

Momma, K. \& Izumi, F. (2011). J. Appl. Cryst. 44, 1272-1276.

Nosyrev, N. A., Treushnikov, E. N. \& Voronkov, A. A. (1976). Dokl. Akad. Nauk SSSR, 231, 1351-1353.

PANalytical (2011). X'Pert Data Collector. PANalytical BV, Almelo, The Netherlands.

Pekov, I. V., Zubkova, N. V., Pushcharovsky, D. Yu., Kolitsch, U. \& Tillmanns, E. (2007). Crystallogr. Rep. 52, 1066-1071.
Petříček, V., Dušek, M. \& Palatinus, L. (2014). Z. Kristallogr. Cryst. Mater. 229, 345-352.

Plaisted, T., Hrma, P., Vienna, J. \& Jiricka, A. (1999). Proceedings of MRS 608, 13-14.

Ramsey, W. G., Bibler, N. E. \& Meaker, T. F. (1995). WM Symposia, Waste Management, 95, 23828-23907.

Voronkov, A. A., Shumyatskaya, N. G. \& Pyatenko, Yu. A. (1970). Zh. Strukt. Khim. 11, 932-933.

Westrip, S. P. (2010). J. Appl. Cryst. 43, 920-925. 


\section{supporting information}

Acta Cryst. (2016). E72, 1434-1437 [https://doi.org/10.1107/S2056989016014225]

\section{Crystal structure of $\mathrm{Na}_{2} \mathrm{HfSi}_{2} \mathrm{O}_{7}$ by Rietveld refinement}

\section{Nicolas Massoni and Pierrick Chevreux}

\section{Computing details}

Data collection: X'Pert Data Collector (PANalytical, 2011); cell refinement: JANA2006 (Petrríček et al., 2014); data reduction: JANA2006 (Petříček et al., 2014); program(s) used to solve structure: JANA2006 (Petříček et al., 2014); program(s) used to refine structure: JANA2006 (Petrríček et al., 2014); molecular graphics: VESTA (Momma \& Izumi, 2011); software used to prepare material for publication: publCIF (Westrip, 2010).

Disodium hafnium disilicate

Crystal data

$\mathrm{Na}_{2} \mathrm{HfSi}_{2} \mathrm{O}_{7}$

$M_{r}=392.6$

Triclinic, $P \overline{1}$

$a=6.6123(2) \AA$

$b=8.7948(3) \AA$

$c=5.41074(15) \AA$

$\alpha=92.603(2)^{\circ}$

$\beta=94.0843(18)^{\circ}$

$\gamma=71.3262(18)^{\circ}$

$V=297.25(2) \AA^{3}$

Data collection

Panalytical XPert MPD Pro diffractometer

Radiation source: sealed X-ray tube

Specimen mounting: packed powder pellet

Refinement

$R_{\mathrm{p}}=0.024$

$R_{\text {wp }}=0.032$

$R_{\text {exp }}=0.015$

$R(F)=0.024$

6423 data points

Profile function: pseudo-Voigt
$Z=2$

$F(000)=356$

$D_{\mathrm{x}}=4.387 \mathrm{Mg} \mathrm{m}^{-3}$

$\mathrm{Cu} K \alpha_{1}$ radiation, $\lambda=1.540562,1.544390 \AA$

$T=293 \mathrm{~K}$

Particle morphology: plate-like

white

flat_sheet, $25 \times 25 \mathrm{~mm}$

Specimen preparation: Prepared at $1393 \mathrm{~K}$ and $100 \mathrm{kPa}$

Data collection mode: reflection

Scan method: step

$2 \theta_{\min }=8.013^{\circ}, 2 \theta_{\max }=120.013^{\circ}, 2 \theta_{\text {step }}=0.017^{\circ}$

67 parameters

0 restraints

6 constraints

Weighting scheme based on measured s.u.'s

$(\Delta / \sigma)_{\max }=0.005$

Background function: Legendre polynoms

Fractional atomic coordinates and isotropic or equivalent isotropic displacement parameters $\left(\hat{A}^{2}\right)$

\begin{tabular}{lllll}
\hline & $x$ & $y$ & $z$ & $U_{\text {iso }} * U_{\text {eq }}$ \\
\hline Na1 & $0.8823(15)$ & $0.0970(12)$ & $0.2630(18)$ & $0.035(4)^{*}$ \\
Na2 & $0.3456(14)$ & $0.5024(10)$ & $0.7608(18)$ & $0.011(3)^{*}$ \\
Hf1 & $0.2880(3)$ & $0.2710(2)$ & $0.2201(3)$ & $0.0158(7)$ \\
Si1 & $0.6528(13)$ & $0.1435(9)$ & $0.7769(13)$ & $0.010(3)^{*}$
\end{tabular}




\begin{tabular}{lllll}
$\mathrm{Si} 2$ & $0.9381(12)$ & $0.3380(8)$ & $0.6861(15)$ & $0.006(2)^{*}$ \\
$\mathrm{O} 1$ & $0.307(2)$ & $0.0382(17)$ & $0.172(3)$ & $0.0030(15)^{*}$ \\
O2 & $0.865(2)$ & $0.1777(14)$ & $0.727(2)$ & $0.0030(15)^{*}$ \\
O3 & $0.494(2)$ & $0.2081(15)$ & $0.530(3)$ & $0.0030(15)^{*}$ \\
O4 & $0.562(2)$ & $0.2522(15)$ & $0.020(3)$ & $0.0030(15)^{*}$ \\
O5 & $-0.003(2)$ & $0.3116(14)$ & $0.401(3)$ & $0.0030(15)^{*}$ \\
O6 & $0.120(2)$ & $0.3300(14)$ & $0.872(3)$ & $0.0030(15)^{*}$ \\
O7 & $0.284(2)$ & $0.5148(14)$ & $0.288(3)$ & $0.0030(15)^{*}$ \\
\hline
\end{tabular}

Atomic displacement parameters $\left(\AA^{2}\right)$

\begin{tabular}{lllllll}
\hline & $U^{11}$ & $U^{22}$ & $U^{33}$ & $U^{12}$ & $U^{13}$ & $U^{23}$ \\
\hline Hf1 & $0.0148(10)$ & $0.0177(11)$ & $0.0126(10)$ & $0.0003(7)$ & $0.0035(7)$ & $0.0111(8)$ \\
\hline
\end{tabular}

Geometric parameters $\left(A,{ }^{o}\right)$

\begin{tabular}{|c|c|c|c|}
\hline $\mathrm{O} 1-\mathrm{Hf1}$ & $2.017(15)$ & $\mathrm{Na} 1-\mathrm{Na} 1^{\mathrm{vii}}$ & $3.169(13)$ \\
\hline $\mathrm{O} 1-\mathrm{Si}^{\mathrm{i}}$ & $1.568(17)$ & $\mathrm{Na} 1-\mathrm{Na} 2^{\mathrm{v}}$ & $3.363(13)$ \\
\hline $\mathrm{O} 2-\mathrm{Si} 1$ & $1.567(18)$ & $\mathrm{Na} 1-\mathrm{Hf} 1^{\text {viii }}$ & $3.513(12)$ \\
\hline $\mathrm{O} 2-\mathrm{Si} 2$ & $1.660(17)$ & $\mathrm{Na} 1-\mathrm{Si} 1^{\mathrm{ii}}$ & $2.919(12)$ \\
\hline $\mathrm{O} 3-\mathrm{Hf1}$ & $2.060(13)$ & $\mathrm{Na} 1-\mathrm{Si} 1$ & $3.210(13)$ \\
\hline $\mathrm{O} 3-\mathrm{Si1}$ & $1.644(15)$ & $\mathrm{Na} 1-\mathrm{Si}^{\mathrm{vii}}$ & $3.140(11)$ \\
\hline $\mathrm{O} 4-\mathrm{Na} 1$ & $2.450(15)$ & $\mathrm{Na} 1-\mathrm{Si} 2$ & $3.132(13)$ \\
\hline $\mathrm{O} 4-\mathrm{Si} 1^{\text {ii }}$ & $1.622(15)$ & $\mathrm{Na} 2-\mathrm{Na}_{2}^{\mathrm{v}}$ & $3.590(14)$ \\
\hline $\mathrm{O} 5-\mathrm{Na} 1^{\mathrm{iii}}$ & $2.323(18)$ & $\mathrm{Na} 2-\mathrm{Na} 2^{\mathrm{ix}}$ & $3.174(13)$ \\
\hline $\mathrm{O} 5-\mathrm{Si} 2^{\mathrm{iii}}$ & $1.605(16)$ & $\mathrm{Na} 2-\mathrm{Hf} 1$ & $3.556(9)$ \\
\hline O6-Hfliv & $2.115(13)$ & $\mathrm{Na} 2-\mathrm{Hf} 1^{\mathrm{iv}}$ & $3.399(10)$ \\
\hline $\mathrm{O} 6-\mathrm{Si} 2^{\mathrm{iii}}$ & $1.497(16)$ & $\mathrm{Na} 2-\mathrm{Hf}^{\mathrm{v}}$ & $3.590(10)$ \\
\hline $\mathrm{O} 7-\mathrm{Na} 2^{\mathrm{v}}$ & $2.441(18)$ & $\mathrm{Na} 2-\mathrm{Si} 1$ & $3.161(10)$ \\
\hline $\mathrm{O} 7-\mathrm{Si}_{2}^{\mathrm{v}}$ & $1.625(13)$ & $\mathrm{Na} 2-\mathrm{Si}_{2} \mathrm{v}$ & $3.052(11)$ \\
\hline $\mathrm{Na} 1-\mathrm{Na} 1^{\mathrm{vi}}$ & $3.453(14)$ & & \\
\hline $\mathrm{Hf} 1-\mathrm{O} 1-\mathrm{Si}^{\mathrm{i}}$ & $161.7(9)$ & $\mathrm{Na} 2^{\mathrm{ix}}-\mathrm{Na} 2-\mathrm{Si} 1$ & $76.2(3)$ \\
\hline $\mathrm{Si} 1-\mathrm{O} 2-\mathrm{Si} 2$ & $136.8(8)$ & $\mathrm{Na} 2^{\mathrm{ix}}-\mathrm{Na} 2-\mathrm{Si} 2^{\mathrm{v}}$ & $153.7(4)$ \\
\hline $\mathrm{Hfl}-\mathrm{O} 3-\mathrm{Si} 1$ & $175.1(10)$ & $\mathrm{Hf} 1-\mathrm{Na} 2-\mathrm{Hfl}^{\mathrm{iv}}$ & $102.1(3)$ \\
\hline $\mathrm{Na} 1-\mathrm{O} 4-\mathrm{Si}^{\mathrm{ii}}$ & $89.2(6)$ & $\mathrm{Hf} 1-\mathrm{Na} 2-\mathrm{Hf} 1^{\mathrm{v}}$ & $119.7(3)$ \\
\hline 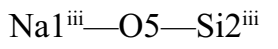 & $104.3(9)$ & $\mathrm{Hf} 1-\mathrm{Na} 2-\mathrm{Si} 1$ & $66.6(2)$ \\
\hline $\mathrm{Hfl} 1^{\mathrm{iv}}-\mathrm{O} 6-\mathrm{Si} 2^{\mathrm{iii}}$ & $153.3(10)$ & $\mathrm{Hf} 1-\mathrm{Na} 2-\mathrm{Si}^{\mathrm{v}}$ & $59.6(2)$ \\
\hline $\mathrm{Na} 2^{\mathrm{v}}-\mathrm{O} 7-\mathrm{Si}^{\mathrm{v}}$ & $134.4(9)$ & $\mathrm{Hf} 1^{\mathrm{iv}}-\mathrm{Na} 2-\mathrm{Hf}^{\mathrm{v}}$ & $126.1(3)$ \\
\hline $\mathrm{O} 4-\mathrm{Na} 1-\mathrm{O} 5^{\mathrm{viii}}$ & $97.2(6)$ & $\mathrm{Hf} \mathrm{I}^{\mathrm{iv}}-\mathrm{Na} 2-\mathrm{Si} 1$ & $62.6(2)$ \\
\hline $\mathrm{O} 4-\mathrm{Na} 1-\mathrm{Na} 1^{\mathrm{vi}}$ & $91.7(4)$ & $\mathrm{Hf} 1^{\mathrm{iv}}-\mathrm{Na} 2-\mathrm{Si}^{\mathrm{v}}{ }^{\mathrm{v}}$ & $134.1(4)$ \\
\hline $\mathrm{O} 4-\mathrm{Na} 1-\mathrm{Na} 1^{\mathrm{vii}}$ & $152.6(6)$ & $\mathrm{Hf} 1 \mathrm{v}-\mathrm{Na} 2-\mathrm{Si} 1$ & $102.9(3)$ \\
\hline $\mathrm{O} 4-\mathrm{Na} 1-\mathrm{Na} 2^{\mathrm{v}}$ & $51.1(4)$ & $\mathrm{Hf} 1^{\mathrm{v}}-\mathrm{Na} 2-\mathrm{Si}^{2}{ }^{\mathrm{v}}$ & $97.7(3)$ \\
\hline $\mathrm{O} 4-\mathrm{Na} 1-\mathrm{Hf} 1^{\text {viii }}$ & $109.2(5)$ & $\mathrm{Si} 1-\mathrm{Na} 2-\mathrm{Si}^{\mathrm{v}}$ & $125.7(4)$ \\
\hline $\mathrm{O} 4-\mathrm{Na} 1-\mathrm{Si}_{1}{ }^{\mathrm{ii}}$ & $33.8(4)$ & $\mathrm{O} 1-\mathrm{Hf} 1-\mathrm{O} 3$ & $88.5(5)$ \\
\hline $\mathrm{O} 4-\mathrm{Na} 1-\mathrm{Si} 1$ & $95.1(5)$ & $\mathrm{O} 1-\mathrm{Hf} 1-\mathrm{O}^{\mathrm{ii}}$ & $91.8(5)$ \\
\hline $\mathrm{O} 4-\mathrm{Na} 1-\mathrm{Si} 1^{\mathrm{vii}}$ & $143.7(5)$ & $\mathrm{O} 1-\mathrm{Hf} 1-\mathrm{Na} 1^{\mathrm{iii}}$ & $51.7(4)$ \\
\hline $\mathrm{O} 4-\mathrm{Na} 1-\mathrm{Si} 2$ & $103.7(5)$ & $\mathrm{O} 1-\mathrm{Hf} 1-\mathrm{Na} 2^{\mathrm{ii}}$ & $123.8(4)$ \\
\hline
\end{tabular}




\begin{tabular}{|c|c|c|c|}
\hline $\mathrm{O} 5^{\mathrm{viii}}-\mathrm{Na} 1-\mathrm{Na} 1^{\mathrm{vi}}$ & $114.1(6)$ & $\mathrm{O} 1-\mathrm{Hf} 1-\mathrm{Na} 2$ & $131.8(4)$ \\
\hline $\mathrm{O} 5^{\mathrm{viii}}-\mathrm{Na} 1-\mathrm{Na} 1^{\mathrm{vii}}$ & $89.9(5)$ & $\mathrm{O} 1-\mathrm{Hf} 1-\mathrm{Na} 2^{\mathrm{v}}$ & $136.4(4)$ \\
\hline $\mathrm{O} 5^{\mathrm{viii}}-\mathrm{Na} 1-\mathrm{Na} 2^{\mathrm{v}}$ & $46.9(4)$ & $\mathrm{O} 3-\mathrm{Hf} 1-\mathrm{O}^{\mathrm{ii}}$ & $171.1(6)$ \\
\hline $\mathrm{O}^{\text {viii }}-\mathrm{Na} 1-\mathrm{Hf} 1^{\text {viii }}$ & $36.4(4)$ & $\mathrm{O} 3-\mathrm{Hf} 1-\mathrm{Na} 1^{\mathrm{iii}}$ & $107.6(5)$ \\
\hline 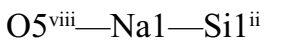 & $113.7(5)$ & $\mathrm{O} 3-\mathrm{Hf} 1-\mathrm{Na} 2^{\mathrm{ii}}$ & $124.6(5)$ \\
\hline $\mathrm{O}^{\mathrm{viii}}-\mathrm{Na} 1-\mathrm{Si} 1$ & $85.7(5)$ & $\mathrm{O} 3-\mathrm{Hf} 1-\mathrm{Na} 2$ & $50.0(4)$ \\
\hline $\mathrm{O} 5^{\mathrm{viii}}-\mathrm{Na} 1-\mathrm{Si}^{\mathrm{vii}}$ & $94.3(5)$ & $\mathrm{O} 3-\mathrm{Hf} 1-\mathrm{Na} 2^{v}$ & $71.4(5)$ \\
\hline $\mathrm{O}^{\text {viii }}-\mathrm{Na} 1-\mathrm{Si} 2$ & $29.8(4)$ & $\mathrm{O} 6^{\mathrm{ii}}-\mathrm{Hf} 1-\mathrm{Na} 1^{\mathrm{iii}}$ & $79.4(4)$ \\
\hline $\mathrm{Na} 1^{\mathrm{vi}}-\mathrm{Na} 1-\mathrm{Na} 1^{\mathrm{vii}}$ & $109.5(3)$ & $\mathrm{O} 6^{\mathrm{ii}}-\mathrm{Hf} 1-\mathrm{Na} 2^{\mathrm{ii}}$ & $48.7(4)$ \\
\hline $\mathrm{Na} 1^{\mathrm{vi}}-\mathrm{Na} 1-\mathrm{Na} 2^{\mathrm{v}}$ & $116.9(4)$ & $\mathrm{O} 6^{\mathrm{ii}}-\mathrm{Hf} 1-\mathrm{Na} 2$ & $133.2(3)$ \\
\hline $\mathrm{Na} 1^{\mathrm{vi}}-\mathrm{Na} 1-\mathrm{Hf} 1^{\text {viii }}$ & $79.2(3)$ & $\mathrm{O} 6^{\mathrm{ii}}-\mathrm{Hf} 1-\mathrm{Na} 2^{\mathrm{v}}$ & $102.6(4)$ \\
\hline $\mathrm{Na} 1^{\mathrm{vi}}-\mathrm{Na} 1-\mathrm{Si}^{\mathrm{ii}}$ & $58.3(3)$ & $\mathrm{Na} 1^{\mathrm{iii}}-\mathrm{Hf} 1-\mathrm{Na} 2^{\mathrm{ii}}$ & $127.8(2)$ \\
\hline 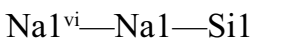 & $158.2(4)$ & 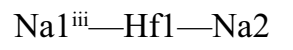 & $111.9(2)$ \\
\hline $\mathrm{Na} 1^{\mathrm{vi}}-\mathrm{Na} 1-\mathrm{Si}^{\mathrm{vii}}$ & $52.3(2)$ & 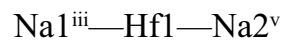 & $171.0(2)$ \\
\hline 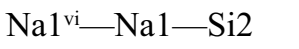 & $141.2(4)$ & $\mathrm{Na} 2^{\mathrm{ii}}-\mathrm{Hf} 1-\mathrm{Na} 2$ & $102.1(2)$ \\
\hline $\mathrm{Na} 1^{\mathrm{vii}}-\mathrm{Na} 1-\mathrm{Na} 2^{\mathrm{v}}$ & $125.8(4)$ & $\mathrm{Na} 2^{\mathrm{ii}}-\mathrm{Hf} 1-\mathrm{Na} 2^{\mathrm{v}}$ & $53.9(2)$ \\
\hline $\mathrm{Na} 1^{\mathrm{vii}}-\mathrm{Na} 1-\mathrm{Hf} 1^{\mathrm{vii}}$ & $92.0(3)$ & $\mathrm{Na} 2-\mathrm{Hf} 1-\mathrm{Na} 2^{\mathrm{v}}$ & $60.3(2)$ \\
\hline $\mathrm{Na} 1^{\mathrm{vii}}-\mathrm{Na} 1-\mathrm{Si} 1^{\mathrm{ii}}$ & $156.1(5)$ & $\mathrm{O} 1{ }^{\mathrm{i}}-\mathrm{Si} 1-\mathrm{O} 2$ & $111.9(8)$ \\
\hline 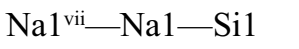 & $59.0(3)$ & $\mathrm{O} 1{ }^{\mathrm{i}}-\mathrm{Si} 1-\mathrm{O} 3$ & $113.6(9)$ \\
\hline $\mathrm{Na} 1^{\mathrm{vii}}-\mathrm{Na} 1-\mathrm{Si}^{\mathrm{vii}}$ & $61.2(3)$ & $\mathrm{O} 1{ }^{\mathrm{i}}-\mathrm{Si} 1-\mathrm{O} 4^{\mathrm{iv}}$ & $109.9(9)$ \\
\hline $\mathrm{Na} 1^{\text {vii_-Na1—Si2 }}$ & $70.6(3)$ & $\mathrm{O} 1{ }^{\mathrm{i}}-\mathrm{Si} 1-\mathrm{Na} 1$ & $97.9(6)$ \\
\hline $\mathrm{Na} 2^{\mathrm{v}}-\mathrm{Na} 1-\mathrm{Hf} 1^{\text {viii }}$ & $71.7(3)$ & $\mathrm{O} 1^{\mathrm{i}}-\mathrm{Si} 1-\mathrm{Na} 1^{\mathrm{iv}}$ & $76.2(6)$ \\
\hline $\mathrm{Na} 2^{\mathrm{v}}-\mathrm{Na} 1-\mathrm{Si} 1^{\mathrm{ii}}$ & $76.5(3)$ & $\mathrm{O} 1^{\mathrm{i}}-\mathrm{Si} 1-\mathrm{Na} 1^{\mathrm{vii}}$ & $61.5(6)$ \\
\hline $\mathrm{Na} 2^{\mathrm{v}}-\mathrm{Na} 1-\mathrm{Si} 1$ & $83.1(3)$ & $\mathrm{O} 1$ - $-\mathrm{Si} 1-\mathrm{Na} 2$ & $150.4(7)$ \\
\hline $\mathrm{Na} 2^{\mathrm{v}}-\mathrm{Na} 1-\mathrm{Si}^{\mathrm{vii}}$ & $135.6(5)$ & $\mathrm{O} 2-\mathrm{Si} 1-\mathrm{O} 3$ & $104.6(8)$ \\
\hline $\mathrm{Na} 2^{\mathrm{v}}-\mathrm{Na} 1-\mathrm{Si} 2$ & $55.9(3)$ & $\mathrm{O} 2-\mathrm{Si} 1-\mathrm{O} 4^{\mathrm{iv}}$ & $105.7(9)$ \\
\hline 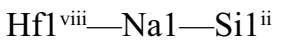 & $104.5(4)$ & $\mathrm{O} 2-\mathrm{Si} 1-\mathrm{Na} 1$ & $52.6(6)$ \\
\hline 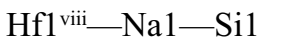 & $117.6(3)$ & $\mathrm{O} 2-\mathrm{Si} 1-\mathrm{Na} 1^{\mathrm{iv}}$ & $77.6(6)$ \\
\hline Hf1 $1^{\text {viii_-Na1-Si1 }} 1^{\text {vii }}$ & $64.0(3)$ & $\mathrm{O} 2-\mathrm{Si} 1-\mathrm{Na} 1^{\mathrm{vii}}$ & $50.5(5)$ \\
\hline $\mathrm{Hf} 1^{\mathrm{viii}}-\mathrm{Na} 1-\mathrm{Si} 2$ & $62.2(3)$ & $\mathrm{O} 2-\mathrm{Si} 1-\mathrm{Na} 2$ & $97.4(6)$ \\
\hline $\mathrm{Si} 1^{\mathrm{ii}}-\mathrm{Na} 1-\mathrm{Si} 1$ & $123.9(4)$ & $\mathrm{O} 3-\mathrm{Si} 1-\mathrm{O} 4^{\mathrm{iv}}$ & $110.8(7)$ \\
\hline $\mathrm{Si} 1^{\mathrm{ii}}-\mathrm{Na} 1-\mathrm{Si}^{\mathrm{vii}}$ & $110.6(4)$ & $\mathrm{O} 3-\mathrm{Si} 1-\mathrm{Na} 1$ & $64.3(6)$ \\
\hline $\mathrm{Si} 1{ }^{\mathrm{ii}}-\mathrm{Na} 1-\mathrm{Si} 2$ & $132.4(4)$ & $\mathrm{O} 3-\mathrm{Si} 1-\mathrm{Na} 1^{\mathrm{iv}}$ & $167.4(6)$ \\
\hline Si1—Na1—Si1 ${ }^{\text {vii }}$ & $120.1(3)$ & $\mathrm{O} 3-\mathrm{Si} 1-\mathrm{Na} 1^{\mathrm{vii}}$ & $121.8(6)$ \\
\hline $\mathrm{Si} 1-\mathrm{Na} 1-\mathrm{Si} 2$ & $56.5(3)$ & $\mathrm{O} 3-\mathrm{Si} 1-\mathrm{Na} 2$ & $59.9(5)$ \\
\hline $\mathrm{Si} 1{ }^{\mathrm{vii}}-\mathrm{Na} 1-\mathrm{Si} 2$ & $103.3(3)$ & $\mathrm{O} 4^{\mathrm{iv}}-\mathrm{Si} 1-\mathrm{Na} 1$ & $150.5(8)$ \\
\hline $\mathrm{O}^{\mathrm{v}}-\mathrm{Na} 2-\mathrm{Na}^{\mathrm{v}}$ & $99.3(5)$ & $\mathrm{O} 4^{\mathrm{iv}}-\mathrm{Si} 1-\mathrm{Na} 1^{\mathrm{iv}}$ & $57.1(5)$ \\
\hline $\mathrm{O} 7^{\mathrm{v}}-\mathrm{Na} 2-\mathrm{Na} 2^{\mathrm{v}}$ & $45.5(4)$ & $\mathrm{O} 4^{\mathrm{iv}}-\mathrm{Si} 1-\mathrm{Na} 1^{\mathrm{vii}}$ & $125.7(6)$ \\
\hline $\mathrm{O}^{\mathrm{v}}-\mathrm{Na} 2-\mathrm{Na} 2^{\mathrm{ix}}$ & $60.6(4)$ & $\mathrm{O} 4^{\mathrm{iv}}-\mathrm{Si} 1-\mathrm{Na} 2$ & $55.6(5)$ \\
\hline $\mathrm{O} 7^{\mathrm{v}}-\mathrm{Na} 2-\mathrm{Hf} 1$ & $96.4(4)$ & $\mathrm{Na} 1-\mathrm{Si} 1-\mathrm{Na} 1^{\mathrm{iv}}$ & $123.9(4)$ \\
\hline $\mathrm{O}^{\mathrm{v}}-\mathrm{Na} 2-\mathrm{Hf}^{\mathrm{iv}}$ & $113.5(4)$ & $\mathrm{Na} 1-\mathrm{Si} 1-\mathrm{Na} 1^{\mathrm{vii}}$ & $59.9(3)$ \\
\hline $\mathrm{O}^{\mathrm{v}}-\mathrm{Na} 2-\mathrm{Hf}^{\mathrm{v}}$ & $35.8(3)$ & $\mathrm{Na} 1-\mathrm{Si} 1-\mathrm{Na} 2$ & $103.3(3)$ \\
\hline $\mathrm{O} 7^{\mathrm{v}}-\mathrm{Na} 2-\mathrm{Si} 1$ & $68.4(4)$ & $\mathrm{Na} 1^{\mathrm{iv}}-\mathrm{Si} 1-\mathrm{Na} 1^{\text {vii }}$ & $69.4(3)$ \\
\hline $\mathrm{O} 7^{\mathrm{v}}-\mathrm{Na} 2-\mathrm{Si}^{\mathrm{v}}$ & $110.4(5)$ & $\mathrm{Na} 1^{\mathrm{iv}}-\mathrm{Si} 1-\mathrm{Na} 2$ & $107.6(3)$ \\
\hline $\mathrm{Na} 1^{\mathrm{v}}-\mathrm{Na} 2-\mathrm{Na} 2^{\mathrm{v}}$ & $91.8(3)$ & $\mathrm{Na} 1 \mathrm{vii}-\mathrm{Si} 1-\mathrm{Na} 2$ & $147.9(4)$ \\
\hline $\mathrm{Na} 1^{\mathrm{v}}-\mathrm{Na} 2-\mathrm{Na} 2^{\mathrm{ix}}$ & $97.3(3)$ & $\mathrm{O} 2-\mathrm{Si} 2-\mathrm{O} 5^{\mathrm{viii}}$ & $100.7(8)$ \\
\hline $\mathrm{Na} 1{ }^{\mathrm{v}}-\mathrm{Na} 2-\mathrm{Hf} 1$ & $117.5(3)$ & $\mathrm{O} 2-\mathrm{Si} 2-\mathrm{O} 6^{\text {viii }}$ & $106.7(8)$ \\
\hline $\mathrm{Na} 1^{\mathrm{v}}-\mathrm{Na} 2-\mathrm{Hf} 1^{\mathrm{iv}}$ & $124.7(3)$ & $\mathrm{O} 2-\mathrm{Si} 2-\mathrm{O}^{\mathrm{v}}$ & $102.8(8)$ \\
\hline
\end{tabular}




\begin{tabular}{|c|c|c|c|}
\hline $\mathrm{Na} 1^{v}-\mathrm{Na} 2-\mathrm{Hfl}^{\mathrm{v}}$ & $64.7(3)$ & $\mathrm{O} 2-\mathrm{Si} 2-\mathrm{Na} 1$ & $55.3(5)$ \\
\hline $\mathrm{Na} 1^{\mathrm{v}}-\mathrm{Na} 2-\mathrm{Si} 1$ & $167.6(4)$ & $\mathrm{O} 2-\mathrm{Si} 2-\mathrm{Na} 2^{\mathrm{v}}$ & $103.7(6)$ \\
\hline $\mathrm{Na} 1^{\mathrm{v}}-\mathrm{Na} 2-\mathrm{Si}_{2}{ }^{\mathrm{v}}$ & $58.2(3)$ & $\mathrm{O}^{\mathrm{viii}}-\mathrm{Si} 2-\mathrm{O}^{\mathrm{viii}}$ & $116.1(9)$ \\
\hline $\mathrm{Na} 2^{\mathrm{v}}-\mathrm{Na} 2-\mathrm{Na} 2^{\mathrm{ix}}$ & $106.1(3)$ & $\mathrm{O}^{\mathrm{viii}}-\mathrm{Si} 2-\mathrm{O}^{\mathrm{v}}$ & $109.8(8)$ \\
\hline $\mathrm{Na} 2^{\mathrm{v}}-\mathrm{Na} 2-\mathrm{Hf} 1$ & $60.3(2)$ & $\mathrm{O} 5$ viii- $\mathrm{Si} 2-\mathrm{Na} 1$ & $46.0(6)$ \\
\hline $\mathrm{Na} 2^{\mathrm{v}}-\mathrm{Na} 2-\mathrm{Hf} 1^{\mathrm{iv}}$ & $142.8(3)$ & $\mathrm{O} 5^{\mathrm{viii}}-\mathrm{Si} 2-\mathrm{Na} 2^{\mathrm{v}}$ & $53.3(5)$ \\
\hline $\mathrm{Na} 2^{\mathrm{v}}-\mathrm{Na} 2-\mathrm{Hfl}^{\mathrm{v}}$ & $59.4(2)$ & $\mathrm{O}^{\mathrm{viii}}-\mathrm{Si} 2-\mathrm{O}^{\mathrm{v}}$ & $118.2(8)$ \\
\hline $\mathrm{Na} 2^{\mathrm{v}}-\mathrm{Na} 2-\mathrm{Si} 1$ & $80.2(3)$ & $\mathrm{O}^{\mathrm{viii}}-\mathrm{Si} 2-\mathrm{Na} 1$ & $131.2(6)$ \\
\hline $\mathrm{Na} 2^{\mathrm{v}}-\mathrm{Na} 2-\mathrm{Si}_{2}{ }^{\mathrm{v}}$ & $68.4(3)$ & $\mathrm{O}^{\mathrm{viii}}-\mathrm{Si} 2-\mathrm{Na} 2^{\mathrm{v}}$ & $149.4(8)$ \\
\hline $\mathrm{Na}{ }^{2 x}-\mathrm{Na} 2-\mathrm{Hf} 1$ & $141.7(3)$ & $\mathrm{O} 7^{\mathrm{v}}-\mathrm{Si} 2-\mathrm{Na} 1$ & $110.3(6)$ \\
\hline $\mathrm{Na} 2^{\mathrm{ix}}-\mathrm{Na} 2-\mathrm{Hf} 1^{\mathrm{iv}}$ & $66.1(3)$ & $\mathrm{O} 7^{\mathrm{v}}-\mathrm{Si} 2-\mathrm{Na} 2^{\mathrm{v}}$ & $57.1(6)$ \\
\hline $\mathrm{Na} 2^{\mathrm{ix}}-\mathrm{Na} 2-\mathrm{Hf}^{\mathrm{v}}$ & $60.0(3)$ & $\mathrm{Na} 1-\mathrm{Si} 2-\mathrm{Na} 2^{\mathrm{v}}$ & $65.9(3)$ \\
\hline
\end{tabular}

Symmetry codes: (i) $-x+1,-y,-z+1$; (ii) $x, y, z-1$; (iii) $x-1, y, z$; (iv) $x, y, z+1$; (v) $-x+1,-y+1,-z+1$; (vi) $-x+2,-y,-z$; (vii) $-x+2,-y,-z+1$; (viii) $x+1, y$, $z$; (ix) $-x+1,-y+1,-z+2$. 